\title{
Captures
}

Figures, théories et pratiques de l'imaginaire

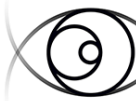

\section{La poésie déambulée : Agnès Varda et Yannick Bellon filment Baudelaire}

\section{Mathilde Labbé}

Volume 2, Number 1, 2017

Écrivains à l'écran

URI: https://id.erudit.org/iderudit/1059805ar

DOI: https://doi.org/10.7202/1059805ar

See table of contents

Publisher(s)

Figura, Centre de recherche sur le texte et l'imaginaire

ISSN

2371-1930 (digital)

Explore this journal

Cite this article

Labbé, M. (2017). La poésie déambulée : Agnès Varda et Yannick Bellon filment Baudelaire. Captures, 2(1). https://doi.org/10.7202/1059805ar
Article abstract

Cet article analyse parallèlement deux films consacrés à Baudelaire : La Plaie et le couteau de Yannick Bellon et Les Dites Cariatides d'Agnès Varda, qui font le choix de contourner les problèmes de figuration du poète en filmant Paris. Leur étude permet de montrer pourquoi la représentation de Baudelaire et de son œuvre constituent une gageure pour les cinéastes, ce qui conduit, à l'écran, à la diffraction du je et au réinvestissement de la ville comme sujet lyrique.
Tous droits réservés () Mathilde Labbé, 2017

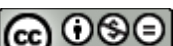

This document is protected by copyright law. Use of the services of Érudit (including reproduction) is subject to its terms and conditions, which can be viewed online.

https://apropos.erudit.org/en/users/policy-on-use/ 
Version enrichie de cet article : http://revuecaptures.org/node/685

\section{La poésie déambulée}

\section{Agnès Varda et Yannick Bellon filment Baudelaire}

\section{Mathilde Labbé}

Résumé :

Cet article analyse parallèlement deux films consacrés à Baudelaire : La Plaie et le couteau de Yannick Bellon et Les Dites Cariatides d'Agnès Varda, qui font le choix de contourner les problèmes de figuration du poète en filmant Paris. Leur étude permet de montrer pourquoi la représentation de Baudelaire et de son œuvre constituent une gageure pour les cinéastes, ce qui conduit, à l'écran, à la diffraction du je et au réinvestissement de la ville comme sujet lyrique.

Si l'écrivain semble plutôt bien représenté au cinéma, Baudelaire dément la règle — c'est d'ailleurs Edgar Poe, alter ego dont l'auteur des Fleurs du Mal fut le traducteur français, qui devient l'un des premiers écrivains cinématographiés (Griffith, 1909). Malgré le potentiel dramatique et tragique du récit de sa vie, rapidement exploité par les biographes 1 , il est si peu représenté au cinéma qu'on pourrait l'en croire absent : même lorsqu'il a été mis en scène ou porté à l'écran, l'accès aux films est parfois difficile, et, parmi les vingt-trois productions qui l'évoquent, nombreuses sont celles qui se contentent de le citer ponctuellement ou de reprendre un terme baudelairien dans leur titre. Rares sont les œuvres cinématographiques pleinement consacrées au poète ou à son œuvre. Trois courts métrages poétiques et quatre documentaires se démarquent cependant de cet ensemble.

La Souriante Madame Beudet de Germaine Dulac (Dulac, 1923), met en scène une lectrice des Fleurs du Mal qui prend conscience de la banalité de sa vie en lisant «La Mort des amants ». L'Invitation au voyage (Dulac, 1927) raconte l'histoire d'une femme qui rencontre un marin dans un cabaret nommé « L'invitation au voyage » et qui rêve que celui-ci la soustrait à la banalité de sa vie. Les deux courts métrages constituent des mises en scène de la lecture dans lesquelles Les Fleurs du Malsont vues à la fois comme la source d'un éveil à soimême et comme le point de départ d'une rêverie déceptive (Labbé: 300-304). Quarante années plus tard, Yannick Bellon réalise une dramatique intitulée La Plaie et le couteau (Bellon, 1967), sur la vie et l'œuvre de Baudelaire. Le film mêle des documents d'époque à des expérimentations cinématographiques autour de poèmes mis en scène. La même année, Max-Pol Fouchet réalise La Nuit et les phares, documentaire diffusé dans le cadre de l'émission Terre des arts et qui participe également de l'évocation scénarisée, tout en favorisant la question de la critique d'art de Baudelaire. II participe par ailleurs au documentaire de Lucien Binot intitulé L'Or et la Boue(1967), dans lequel interviennent également Pierre de Boisdeffre, Pierre 
Emmanuel, Claude Pichois, Claude Roy et Jean Tordeur. Sept ans plus tard, Alain Bosquet et Dominique Delouche réalisent un long métrage intitulé Opéra pour Baudelaire (Bosquet et Delouche, 1974). Moins historique et plus onirique que le film de Bellon, il ne sera cité ici qu'à titre de comparaison pour replacer ce dernier dans le style télévisuel de son époque. Enfin, Les Dites Cariatides d'Agnès Varda (1984), court métrage quasi documentaire à la première personne sur ces héroïnes de l'architecture haussmannienne, fait une place importante aux Fleurs du Mal, si bien qu'il semble devenir, en cours de propos, un film sur la poésie de Baudelaire.

Le choix d'aborder l'image de Baudelaire au cinéma à travers le film de Yannick Bellon et celui d'Agnès Varda se justifie par une certaine parenté esthétique entre ces deux œuvres, qui participent chacune à leur manière d'un renouvellement des formes cinématographiques et télévisuelles, mais surtout par une communauté d'approche de la poésie baudelairienne, représentée par le biais de la ville. Chacun des deux films constitue en effet une déambulation littéraire dans les « plis sinueux » (Baudelaire, 1861: 89) de la ville, et donne à voir Paris de jour comme de nuit, en faisant alterner le point de vue du marcheur et les vues surplombantes. Ils soulèvent à ce titre plusieurs questions, qui tiennent toutes à la figuration de l'écrivain à l'écran en régime documentaire 2 . La première concerne le statut de l'évocation par opposition à l'incarnation dans un cadre documentariste. Une telle approche de la figure de l'écrivain n'exclut pas toujours la fiction et, bien que l'on puisse s'attendre, à l'ère du nouveau roman et de la nouvelle vague, à une approche critique de la biographie, il y a bien fabrication d'une image de l'écrivain. II convient par ailleurs de se demander si la poésie constitue un cas particulier dans la représentation de l'écrivain à l'écran, et pourquoi la ville joue un rôle si important dans la figuration du poète à cette époque et dans ces films. Enfin, il faut s'interroger sur la possibilité de penser un cinéma baudelairien. Je m'intéresserai ainsi à la promotion de la ville au rang de personnage dans ces films, qui témoignent de deux fictions complémentaires de l'auteur, et qui tentent, chacun à leur manière, de transposer à l'écran l'esthétique baudelairienne.

\section{Filmer la ville}

Figurer l'écriture poétique et le personnage du poète au cinéma n'est pas chose aisée, sauf si le poète en question a précisément consacré son œuvre à une « capitale infâme » (Baudelaire, 1861: 191), où il va « [s]'exercer seul à [s]a fantasque escrime » (1857: 83). Dans La Plaie et le couteau de Yannick Bellon comme dans Les Dites Cariatides d'Agnès Varda, il semble ainsi que filmer Baudelaire revienne à filmer Paris. Le paysage parisien est à même de donner corps à la poésie de Baudelaire et permet de mettre en scène, outre son lieu de vie, son objet et son regard d'artiste.

Comme Baudelaire fait de Paris une clé de sa poétique, les films de Yannick Bellon, d'Alain Bosquet et d'Agnès Varda s'emparent de ce sujet baudelairien et semblent parfois constituer des réécritures de ces vers 
du «Cygne » : «Le vieux Paris n'est plus (la forme d'une ville / Change plus vite hélas que le cœur d'un mortel) » (1861: 85). Walter Benjamin, dans ses essais, et Aragon après lui, dans sa poésie, ont contribué à ériger Baudelaire en poète de la ville (Benjamin, 1938; Aragon, 1957). Paris change, de même, dans le court métrage d'Agnès Varda, qui s'attache à la décennie 1860-1870, pendant laquelle les cariatides et les atlantes (leur équivalent masculin) fleurissent sur les façades parisiennes. La ville, dans son film, est présentée sous l'angle du vestige et de la trace, comme un répertoire de signes dont on aurait perdu le sens : la cariatide de Turbigo, appelée « ange du bizarre » (Varda: 10:23), se dresse dans sa rigueur impénétrable et mystérieuse. Les prises de vues architecturales alternent avec des séquences consacrées au mouvement contemporain des citadins et des omnibus. La couleur des vêtements années 80 contraste avec les tons ternes, de gris-ocre et de noir, des statues. Les métamorphoses de la ville sont ainsi mises en évidence par le choix d'objets symboliques de la modernité technique ou de paysages caractéristiques. Alain Bosquet et Dominique Delouche vont plus loin encore dans l'anachronisme en superposant le souvenir des travaux haussmanniens aux grands travaux de l'époque pompidolienne (Bosquet, 1974: 0:42-0:46).

Objet poétique, la ville est également lieu de la création. Chez Agnès Varda et chez Yannick Bellon, elle apparaît même comme le lieu propre de la poésie. Le travail poétique est ici affaire de déambulation : tout comme le poète du «Soleil » va «Flairant dans tous les coins les hasards de la rime, / Trébuchant sur les mots comme sur les pavés / Heurtant parfois des vers depuis longtemps rêvés ", Jacques Roubaud, qui l'incarne dans La Plaie et le couteau, dit écrire en marchant (Roubaud, 2013). C'est pourquoi le travail poétique n'est ici figuré ni par l'angoisse de la page blanche, ni par la posture ou l'hexis littéraire (Durand, 2014) de l'écrivain à sa table de travail, mais à travers la flânerie. Ces deux films semblent ainsi répondre à la déclaration d'Henri Dutilleux expliquant pourquoi il a dû renoncer à son projet de mise en scène des Fleurs du $\mathrm{Mal}$ avec Roland Petit : « Je pensais qu'il fallait prendre une certaine distance avec les poèmes de Baudelaire [...] qu'il était très difficile de le représenter en scène, de le voir, qu'avec Baudelaire on avait besoin vraiment d'un grand espace » (Nectoux, 2008). Agnès Varda, dont le film évoque à la fois la vie et l'œuvre de Baudelaire, projette ces poèmes, par le biais de la voix hors champ, sur l'écran de paysages entiers.

La transposition de la poétique baudelairienne à l'écran ne fait pas seulement appel aux paysages parisiens mais aussi à la foule qui les peuple. Celle-ci permet à Yannick Bellon comme à Agnès Varda de mettre en scène le parcours du rôdeur parisien dans la ville, et, à travers le « bain de multitude » (Baudelaire, 1869: 291), la dépersonnalisation du sujet lyrique élevée au rang de principe esthétique par Baudelaire dans « Les Foules ». Le promeneur, au sein de la multitude, jouit d'un anonymat qui convient bien au lyrisme baudelairien : le poète est tour à tour " lui-même et autrui. Comme ces âmes errantes qui cherchent un corps, il entre, quand il veut, dans le personnage de chacun » (Baudelaire, 1869: 291). La « fourmillante cité » donne ainsi aux poèmes et à la vie de Baudelaire un cadre qui permet d'en faire des objets cinématographiques. La 
foule constitue en effet le point de contact entre la modernité baudelairienne et la modernité des frères Lumière (Lumière, 1895). Yannick Bellon tire parti de cet objet, qui est tout à la fois thème et principe esthétique, dans plusieurs passages de son film.

La récitation des «Foules » se superpose dans La Plaie et le couteauà des images dont l'ancrage historique est quelque peu atténué par l'usage noir et blanc — malgré le passage de quelques voitures à moteur au second plan et la modernité des costumes entrevus dans la foule - et invite à voir dans le visage de Jacques Roubaud, alors âgé de trente-cinq ans, un reflet de celui de Baudelaire immortalisé par Carjat un siècle plus tôt (1861-1862). Cependant, une telle incarnation reste exceptionnelle dans le film de Yannick Bellon. En effet, la réalisatrice procède plutôt à une dépersonnalisation visuelle du sujet lyrique en diffractant l'image de Baudelaire. Agnès Varda, pour sa part, fait simplement l'ellipse de ce portrait. Le choix de ces deux opérations contradictoires s'explique par le fait que les deux films adoptent deux approches différentes du rapport entre l'homme et l'œuvre, bien que toutes deux privilégient la seconde sur le premier.

\section{Deux fictions de l'auteur}

La dramatique de Yannick Bellon et le documentaire à la première personne d'Agnès Varda se concentrent sur l'œuvre de Baudelaire et n'accordent à la biographie de l'auteur qu'une part réduite. Cependant, dans le contexte de l'ère du soupçon et des lectures textualistes, ces deux approches se distinguent par une actualisation de l'œuvre à travers des fictions de l'auteur. La Plaie et le couteause présente comme un documentaire sur l'œuvre de Baudelaire et bouleverse dès les premières minutes la chronologie de la vie du poète en évoquant d'abord sa mort. Ce premier moment est suivi par un examen du procès des Fleurs du Mal, et le documentaire se clôt, au terme d'un parcours presque à rebours, par l'évocation successive du Salon de 1846 et du séjour de Baudelaire en Belgique (1864-1866). Les documents historiques et biographiques présentés dans le film ne sont en effet convoqués que pour appuyer l'analyse de l'œuvre ou son actualisation. Yannick Bellon use de ces éléments de contexte historique pour introduire des mises en scène de poèmes. Les glissements de l'historique à l'onirique, et inversement, sont donc constants. L'on passe d'une mise en scène du poème «À une heure du matin » à la correspondance de Baudelaire avec sa mère (Bellon: 58:3358:43) sans changer d'acteur ni de cadre : c'est alors bien le poète qui est représenté, allongé sur son divan, parlant pour lui-même à Caroline Aupick.

La fiction d'auteur créée par Yannick Bellon se démarque de ce que l'on appellerait aujourd'hui unbiopic dans la mesure où elle est destinée à faire vivre l'œuvre plus qu'à faire revivre l'homme. Par la multiplication des acteurs, Yannick Bellon choisit d'empêcher l'identification du poète à un visage ${ }^{3}$. Certes, le générique annonce le poète Jacques Roubaud dans le rôle de Baudelaire. Ce choix s'explique, selon l'acteur, par une ressemblance entre le dernier Baudelaire et son propre visage à trente-cinq ans : « j'avais un petit peu la tête 
de Baudelaire sur les dernières photos » (Roubaud, 2013)4. Cependant, plusieurs acteurs se relaient. Après la mise en scène d' "À une heure du matin », dans laquelle Baudelaire est représenté sous les traits de JeanFrançois Adam, intervient celle du «Flacon », pour laquelle le poète a pour visage celui de Jacques Roubaud.

D'autres les précèdent ou leur succèdent dans des séquences scénarisées comparables («Le Balcon », récité par Serge Reggiani dans un décor extérieur nocturne et arboré; " Une charogne », poème déclamé par Serge Merlin dans le décor d'un café) ou à l'occasion de récitations théâtrales plus attendues («Le Léthé » dit par Michel Simon, «Enivrez-vous » par celle de Laurent Terzieff). Cette figuration multiple du poète n'a pas vocation à constituer une représentation cohérente : La Plaie et le couteaumet davantage en scène la fonction ou la posture de poète que l'homme Baudelaire.

Plutôt que la diffraction de l'image du poète, Agnès Varda fait le choix de l'ellipse. La fiction d'auteur qui se dégage des Dites Cariatides ne passe pas par l'identification de Baudelaire à un portrait visuel, mais par une évocation toute personnelle du poète, dans laquelle la réalisatrice lui rend hommage :

Baudelaire courtise Madame Sabatier depuis des années. II lui a envoyé des poèmes, anonymes d'abord. Et puis, un soir, après une séance du procès suscité par la parution des Fleurs du Mal, Madame Sabatier se donne au poète. Le lendemain, il lui écrit une lettre de rupture : « il y a quelques jours, tu étais une divinité, ce qui est si commode, ce qui est si beau, si inviolable. Te voilà femme maintenant. » On en pleurerait; mais je pleure encore plus sur les deux dernières années de Baudelaire. En 1866, il a quarante-cinq ans, il est célèbre mais pauvre, usé, aigri, malade. II tombe dans une église et reste hémiplégique et aphasique. II est muet. II arrive quand même à articuler " crénom de crénom! » Dix-huit mois plus tard, il meurt, toujours muet. (Varda: 7:55-8:53.)

Si c'est la poésie de Baudelaire qui est à l'honneur, l'évocation du destin tragique du poète provoque chez la narratrice un sentiment de compassion. Même lorsqu'il s'agit de commenter les poèmes, la dynamique de l'émotion personnelle est à l'œuvre : pour Agnès Varda, Baudelaire est le « Poète des poètes, celui qui a chanté la douleur et les femmes comme personne » (Varda: 6:25-6:29). Sans mettre en scène la vie de Baudelaire, la réalisatrice reprend à son compte la souffrance du poète.

D'une manière générale, l'un et l'autre film privilégient l'œuvre et non l'homme, et semblent constituer, plutôt que des documentaires ou des reportages, des transpositions de l'esthétique baudelairienne à l'écran.

\section{Transpositions cinématographiques de l'esthétique baudelairienne}

II n'est pas question, pour Yannick Bellon ou pour Agnès Varda, de dresser à proprement parler un portrait de Baudelaire. L'évocation de son œuvre ne sert pas un propos documentaire mais un dialogue esthétique avec le 
poète, qui passe par une tentative d'application de sa poétique au support filmique. En ce sens, il s'agit bien d'expérimentations esthétiques dont la dimension pseudo-biographique n'est qu'une entrée en matière. II faut ainsi reconnaître à ces deux films une ambition particulière, qui en fait des œuvres critiques.

Yannick Bellon fait de la matière poétique la base de son travail cinématographique en adaptant à l'écran des poèmes entiers. Dans ce cadre, elle fait une place toute particulière aux poèmes en prose du Spleen de Paris, exploitant tantôt la dimension apologétique («Portraits de maîtresses »), tantôt la veine moraliste (« Les Foules »), tantôt la tonalité intimiste ("À une heure du matin ») du recueil. Si l'œuvre de Baudelaire avait déjà été évoquée à l'écran par Dulac, le film de Yannick Bellon constitue sa première mise en images, le premier exemple d'une tentative d'association de la modernité baudelairienne à la modernité cinématographique à travers des mises en scène de poèmes.

Cette transposition repose sur l'élaboration d'outils cinématographiques spécifiques. Yannick Bellon utilise ainsi une alternance de documents et de mises en scène pour exposer aux spectateurs l'esthétique du poète. L'une des dernières séquences du film vise à définir la modernité de Baudelaire : des gravures représentant la ville sont montrées à l'écran pendant que le commentaire explicite le désir du poète de saisir la beauté moderne et de tirer l'éternel du transitoire. Yannick Bellon fait suivre cet exposé théorique d'une interprétation du poème «À une passante », dans laquelle elle s'efforce de traduire l'esthétique baudelairienne par un travail de décomposition du mouvement : l'image cinématographique s'y trouve scindée en photogrammes.

La femme, en grand deuil, est arrêtée dans la course de son tapis roulant, élément qui actualise l'idée de modernité technique dans le contexte des années 60 . Elle est alors saisie par plusieurs images fixes dans une expression et une pose qui font d'elle une madone et révèlent, comme les vers de Baudelaire « les stigmates que l'amour lui-même reçoit de la grande ville ", selon l'expression de Benjamin (1938: 72). Cette séquence est immédiatement suivie d'une série d'images plus énigmatiques : plan fixe sur une vitrine dans laquelle se reflète le monde extérieur, vue de la Seine embrumée, gros plan sur un portrait de Baudelaire par Nadar.

D’une manière générale, La Plaie et le couteau donne à voir une traduction cinématographique du spleen qui, « despotique », « plante son drapeau noir » sur le « crâne incliné » du poète (Baudelaire, 1857: 75). Un motif cinématographique rythme en effet le film : la déambulation est ponctuée par la répétition de certaines prises de vue, si bien que, loin de constituer une marche au hasard, elle apparaît plutôt comme un parcours obsessionnel, contraint par des limites horizontales et verticales. On songe, en voyant ces images, au Procès d'Orson Welles sorti cinq ans plus tôt, et en particulier à la scène dans laquelle Joseph K., sortant de chez le peintre, tente de s'enfuir par une série de couloirs et d'étroites ruelles. Le film de Yannick Bellon partage avec celui de Welles non seulement une certaine esthétique du contraste - soutenu ici par la musique entêtante d'Enrique Belloch — , mais aussi une dynamique narrative implacable qui conduit à la mort. Les images 
obsédantes du poète arpentant Paris à travers la même unique ruelle rectiligne inscrivent le film dans un style d'époque — imitation d'un expressionnisme déjà ancien — et offrent une actualisation de l'angoisse baudelairienne propre à l'univers cinématographique.

Cette errance obstinée, esquisse d'une esthétique de l'obsession, trouve un équivalent chez Varda dans un mouvement répété de la caméra. Les Dites Cariatides, en effet, sont très souvent filmées au moyen d'un travelling ascendant qui révèle progressivement les formes féminines puis la tête de la statue, laquelle crée toujours la surprise : détachement, affliction, rigidité, masque. L'apparition du visage ponctue le discours de la voix off, lequel se présente comme une enquête sur la question de la beauté. L'histoire des Cariatides, femmes devenues butin de guerre, que l'on fit défiler « avec leurs belles robes et leurs ornements » (Varda: 2:45-2:47) et qui devinrent le modèle de colonnes célébrant cette victoire, conduit Agnès Varda à s'interroger sur la manière dont le visage des statues exprime l'effort physique : l'expression des atlantes révèle la tension des muscles, tandis que les cariatides portent leur charge «l'air de rien » (5:11). L'histoire de ces colonnes humaines soulève trois questions dans le discours que la réalisatrice tient hors champ. Celle-ci s'intéresse au rôle d'une histoire violente dans la formation d'un canon esthétique, mais aussi à la question de la représentation différenciée des hommes et des femmes - force brute ou beauté impénétrable -, et au sens du geste représenté. Agnès Varda juxtapose ainsi des vues de cariatides avec des images contemporaines de livreurs (04:35). Ceux-ci portent sur leurs épaules des chargements destinés aux habitants des immeubles dont celles-là ornent les façades, dominant l'activité humaine. La verticalité des statues est ainsi redoublée par l'organisation des plans et les déplacements de la caméra : des prises de vue vertigineuses transportent le spectateur du trottoir au balcon et retour, pour le mener finalement jusqu'au ciel, par-dessus les toits (Varda, 1984: 10:50).

C'est en cela que les cariatides apparaissent in fine comme le prétexte d'un film poétique sur la conception baudelairienne de la beauté $\underline{5}$. Cette beauté double, platonicienne et meurtrière d'une part, moderne et mouvante, d'autre part, est patiemment revisitée par Agnès Varda. En superposant la récitation aux images des cariatides, la réalisatrice rend aux poèmes de Baudelaire leur inspiration sculpturale $\underline{6}$. L'usage répété du travelling ascendant sur les statues, parfois recommencé en début de strophe, peut être envisagé à la fois comme une manière de mettre en évidence le principe de l'écriture versifiée ou comme une démonstration, par la répétition, de l'échec éternel des poètes qui, tentant de saisir la beauté, « consum[ent] leurs jours en d'austères études » (Baudelaire, 1857: 21).

L'analyse des modalités de figuration du poète à l'écran invite à une réflexion sur le genre cinématographique adopté dans ces deux films, que l'on peut hésiter à classer : s'agit-il de dramatiques, de documentaires, 
de reportages, de biographies filmées ou de mises en scène de poèmes? Si l'un et l'autre apparaissent d'abord comme des reportages, la dynamique qui détermine leur composition se révèle plutôt être celle, audacieuse, de la mise en voix et en images de textes poétiques, celle de l'évocation scénarisée. L'actualisation, dans sa dimension volontairement anachronique, est la composante majeure des deux films. L'aspect documentaire de celui de Yannick Bellon reste secondaire et pourrait n'être qu'un moyen commode de ménager des transitions entre les mises en scène de poèmes. On observe ainsi un glissement de la représentation de l'écrivain à la mise en scène de son œuvre, dans le film de Yannick Bellon, et un glissement du reportage sur la ville à une actualisation de la poésie baudelairienne, dans celui d’Agnès Varda.

Les images de Baudelaire qui résultent de ces deux évocations sont deux figures contrastées. Yannick Bellon propose un Baudelaire spleenétique et misanthrope, qui jouit de la foule mais s'en garde et navigue constamment entre le plaisir de voir son semblable et l'horreur d'une «tyrannie de la face humaine » (Baudelaire, 1869: 287). Le corps de l'écrivain arpentant la ville devient peu à peu le centre du film. Agnès Varda compose, quant à elle, un Baudelaire lumineux, dont elle explore cependant aussi la part d'ombre, qui va de l'attrait pour un impossible idéal à la curiosité suscitée par la maladie et la mort.

La poésie constitue un cas particulier dans la représentation de l'écrivain à l'écran, dans la mesure où elle n'est pas seulement art de l'écrit, mais aussi spectacle, quand elle est déclamée. De plus, l'adaptation de l'œuvre poétique n'est pas l'adaptation du roman : il ne peut s'agir, en l'espèce, de faire appel à la dimension narrative du cinéma. Ceci explique peut-être que peu de cinéastes aient tenté de porter la poésie baudelairienne à l'écran. Les deux œuvres qui nous intéressent constituent ainsi de belles exceptions. Alors que les films impliquant l'œuvre de Baudelaire se sont souvent limités à une représentation des conséquences de sa lecture (Dulac, 1923; Dulac, 1927), Yannick Bellon et Agnès Varda ont fait le pari d'un cinéma baudelairien non narratif, abstrait et poétique, donnant ainsi la preuve du fait qu'un échange esthétique fécond est possible.

1. Il existe plus d'une soixantaine de textes littéraires biographiques ou à orientation biographique consacrés à Baudelaire. Pour la liste complète de ces ouvrages, voir Labbé (2014: 418-422).

2. Les deux films s'annoncent comme des reportages mais relèvent plutôt du documentaire et mêlent document et fiction dans la figuration de l'écrivain.

3. Seul Jean-Pierre Rawson se risque véritablement à une figuration de Baudelaire à travers un acteur unique, dans son film de 1991. C'est alors Antoine Duléry qui incarne le poète.

4. Avoir " un petit peu la tête de Baudelaire ", c'est ici posséder le même mouvement de mèche sur le front, mais aussi le regard profond et vif et la lèvre inférieure pleine de dédain que l'on observe respectivement dans le portrait photographique réalisé par Carjat en 1861 ou 1862 et dans les autoportraits de Baudelaire. Ce visage est peu à peu devenu la représentation canonique de Baudelaire, probablement parce que la photographie, précisément conforme à la description que Baudelaire fait de lui-même en marge de ses autoportraits, et aujourd'hui libre de droits, a très souvent été reproduite en couverture d'ouvrages consacrés au 
poète. C'est à travers ce portrait également que Baudelaire a été identifié dans la photographie où il apparaît derrière Félix Arnauldet immortalisé par Carjat (Plantureux, 2013).

5. D'autres allusions à Baudelaire marquent le goût de Varda pour la poésie de Baudelaire, de Jacquot de Nantes à Cléo de 5 à 7 (Labbé, 2014: 295-299).

6. “Le Masque » et « Danse Macabre », dédiés à Ernest Christophe, peuvent être compris comme des ekphrasis (Guégan, 2003).

\section{Bibliographie}

ArAgon, Louis. 1957. «Plein feu ». Les Lettres françaises, 12-18 décembre, p. 8.

Benjamin, Walter. 2002 [1938]. Le Paris du Second Empire chez Baudelaire, traduction et préface par Jean Lacoste. Paris : Payot.

Baudelaire, Charles. 1975 [1857/1861]. «Les Fleurs du Mal », dans CEuvres complètes, texte établi, présenté et annoté par Claude Pichois. Paris : Gallimard, «Bibliothèque de la Pléiade », t. i, p. 1-134.

Baudelaire, Charles. 1975 [1869]. «Le Spleen de Paris (Petits poëmes en prose)», dans CEuvres complètes, texte établi, présenté et annoté par Claude Pichois. Paris : Gallimard, « Bibliothèque de la Pléiade », t. I, p. 273-363.

Bellon, Yannick (réal.). 1967. La Plaie et le couteau France : Première Chaîne, 54 min.

Binot, Lucien (réal.). 1967. L'Or et la boue. Belgique : RTBF, 43 min.

CarJat, Étienne. [s. d.]. Baudelaire, photographie. Paris : Bibliothèque historique de la ville de Paris, $23 \times 18$ $\mathrm{cm}$.

Conen, Nadja. 2013. Les poètes modernes et le cinéma (1910-1930). Paris : Classiques Garnier, 449 p.

Bosquet, Alain et Dominique Delouche (réal.). 1974. Opéra pour Baudelaire. France : Deuxième Chaîne, 52 min.

DULAC, Germaine (réal.). 1927. L’Invitation au voyage. $36 \mathrm{~min}$.

DuLAC, Germaine (réal.). 1923. La Souriante Madame Beudet. France : Colisée Films, 54 min.

DuRAND, Pascal. 2014. «De Nadar à Dornac. Hexis corporelle et portrait photographique de l'écrivain». COnTEXTES, no 14. <https://journals.openedition.org/contextes/5933>.

Fouchet, Max-Pol. 2001. Baudelaire annoncait l'art d'aujourd'hui. Roubaix : La Renaissance du livre.

FOUCHET, Max-Pol. 1968. «Un prophète de l'art d'aujourd'hui ». Les Nouvelles littéraires, 21, novembre, p. 18.

Fouchet, Max-Pol et Ginette GuitARd-Auviste. 1957. «Comment je vois Baudelaire ». Les Nouvelles littéraires, 16, novembre, p. 8.

FOUCHET, Max-Pol (réal.). 1967. La Nuit et les phares. Baudelaire critique d'art France : La Première chaîne; ORTF, «Terre des arts», $85 \mathrm{~min}$.

GuÉGAN, Stéphane. 2003. «À propos d'Ernest Christophe. D’une allégorie l'autre », dans André GuYAux et 
Bertrand Marchal (dir.), Les Fleurs du Mal. Actes du colloque de la Sorbonne, 10 et 11 janvier 2003 Paris : Presses de l'Université Paris-Sorbonne, p. 95-106.

GrifFITH, David W (réal.). 1909. Edgar Allen Poe, "American Mutoscope \& Biograph". États-Unis : Studio City, $9 \mathrm{~min}$.

RouBAUD, Jacques. 2013. «Entretien non publié », propos recueillis par Mathilde Labbé.

LABBÉ, Mathilde. 2014. «Héritages baudelairiens. 1931-2013 ». Thèse de doctorat. Université ParisSorbonne, $590 \mathrm{f}$.

Martens, David et Myriam Watthee-Delmotte (dir.). 2012. L'Écrivain, un objet culturel. Dijon : Éditions universitaires de Dijon, $327 \mathrm{p}$.

Nectoux, Jean-Michel. 2008. «Un projet de ballet », entretien avec Henri Dutilleux, dans Henri Dutilleux et André CAPLET (dir.), Tout un monde lointain et Trois strophes sur le nom de Sacher; Épiphanie Paris : Aeon, CD, 2 min $25 \mathrm{sec}$.

VARDA, Agnès (réal.). 1984. Les Dites Cariatides. France : Ciné-Tamaris, 12 min. 